\section{Diagnostic and Therapeutic Uses of A Novel Metallic Conduits Set and Fiber Optic Bronchoscope in Undiagnosed Pleural Diseases}

\author{
Hanmant Ganpati Varudkar*, Swapnil Jain and Arti Julka \\ Department of Pulmonary Medicine, Gardi Medical College, Ujjain, Madhya \\ Pradesh, India
}

\begin{abstract}
\section{Back ground}

Since thoracoscopy needs special instruments, training and higher cost it is sparingly used modality in our country. We have designed and manufactured a specialized set of metallic conduits to facilitate the use of Fiber Optic Bronchoscope (FOB) as thoracoscope (Patent Application No 1066/MUM/2012 published on14/12/2012; and Application No 1400/MUM/2012 published on16/11/12). (Fog No 1). Here is the report of these procedures performed in bronchoscopy rooms.
\end{abstract}

\section{Materials and methods}

All cases of moderate to massive pleural effusions, pneumothoraces, or hydropneuthoraces were fully investigated to clinch the diagnosis; but undiagnosed cases were included in this study. These patients were subjected thoracoscopy/ pleuroscopy through chest stoma in suitable site. First a simple straight conduit was used for draining the contents in pleural cavity. Then it was removed, and the short curved conduit was passed through the stoma, and Fiber optic bronchoscope was negotiated through it to visualize parietal pleura. Then the conduit was replaced with parietal pleuroscopy conduit which facilitates inspection of parietal pleura fully. Then visceral pleuroscopy conduit was used for doing inspection of visceral pleura.

*Corresponding author: Hanmant Ganpati Varudkar, Department of Pulmonary Medicine, Gardi Medical College, Ujjain, Madhya Pradesh, India, Tel: +91 9893807378; E-mail: hgvarud@gmail.com

Citation: Varudkar HG, Jain S, Julka A (2020) Diagnostic and Therapeutic Uses of A Novel Metallic Conduits Set and Fiber Optic Bronchoscope in Undiagnosed Pleural Diseases. J Pulm Med Respir Res 6: 045.

Received: August 06, 2020; Accepted: October 27, 2020; Published: November 03, 2020

Copyright: () 2020 Varudkar HG, et al. This is an open-access article distributed under the terms of the Creative Commons Attribution License, which permits unrestricted use, distribution and reproduction in any medium, provided the original author and source are credited.
Diagnostic procedures like biopsy, and therapeutic interventions like adhesiolysis, retrieval of foreign body, pleurodesis were done. FOB and onduits were removed; chest-tube -drain was kept until full lung expansion and then chest tubes removed.

\section{Results}

Out of 121 patients with pleural effusions, hydropneuthorax or hemothorax we could clinch diagnosis in 67 patients with routine investigations; but remaining 54 patients with undetermined diagnoses were subjected to pleuroscopy with our technique. We could diagnose 21 cases of tuberculosis, 15 cases of malignancies. More over adhesiolysis in 3 cases, repositioning of chest tube in 2, Pleurodesis in 5 , and foreign body retrieval in one case was done. There were significant complications.

\section{Conclusion}

Our pleuroscopy conduits have greatly facilitated the use of Fiber optic bronchoscope as thoracoscope in diagnosis and therapeutics of pleural diseases with acceptable complications.

Keywords: Fiber optic bronchoscope; Pleuroscopy; Pneumothorax; Pleural diseases; Thoracoscopy

\section{Introduction}

Due to industrialization, pollution and smoking chronic respiratory diseases are on rise in India. Thus not only tuberculosis but also diseases like cancers, bacterial empyema are abundant in our country. Needless to say those acute as well chronic pleural diseases need correct diagnosis and management.

In most of the cases exact diagnosis of pleural pathology remains a matter of conjecture. Clinician's acumen, radiology and some laboratory tests which are available in the concerned set up are used if patient is affording type. If not then management is started on empirical basis; and this may culminate into irreparable physical damage, financial loss and social burden. Some simple therapeutic interventions are not done by the physicians. Breaking adhesions, achieving good pleurodesis and adhesiolysis is deferred by chest physicians due to lack of easy and safe techniques. This also adds to the morbidity and physical incapacity of the patients. If easy and economical solutions are available to our physicians they will treat their patients more rationally.

Retrieval of foreign bodies from pleura is another major difficult task in our country. Their removal is possible only in urban modern centers where all the facilities are available. Thus we need some devices which will enable easy retrieval of foreign bodies. Now a day, there are many more young doctors interested in respiratory specialty. They require fiber optic bronchoscope for their clinical work primarily. However additional thoracoscopy is a heavy economical burden. They will be happy if their own fiber optic bronchoscope could be used for diagnosis of pleural pathology.

For this purpose we have conventional thoracoscope, Video Assisted Thoracoscope (VATS) in vogue. These are highly sophisticated costly scopes not manufactured by any Indian company. This procedure requires major operation theater which increases cost in turn. Many a times we need general anesthesia which adds its cost and 
Citation: Varudkar HG, Jain S, Julka A (2020) Diagnostic and Therapeutic Uses of A Novel Metallic Conduits Set and Fiber Optic Bronchoscope in Undiagnosed Pleural Diseases. J Pulm Med Respir Res 6: 045.

complications. All these factors restrict utility of such an essential procedure to only limited sophisticated institutions.

There are many substituting equipments for thoracoscopies for instance rigid bronchoscope. It is straight metallic rigid tube based on simple optics. Therefore it cannot cover larger pleural surface, and it also cannot give access to difficult pleural spaces. It cannot visualize large parietal pleural due to rigidity. It cannot visualize diaphragmatic pleural surface neither it can visualize intra fissural pleural surface. It cannot help in adhesiolysis, ICD tube placement, or pleurodesis. Flexible fiber optic bronchoscope, basically, is designed for tubular structures like tracheobronchial tree. However people have used only this instrument for pleuroscopy alone or with the help of a readily available conduits. Due to flexibility its tip cannot be manipulated in a desired direction because the pleural space is not a tubular. Therefore only this instrument has limited role in thoracoscopy or pleuroscopy.

Some workers have passed FOB through rigid tubes like laryngoscope with limited success. Again they have achieved limited success and acceptance. All these procedures have not received universal acceptance because right reasons. Thus there is a yawning gap between the requirements of our chest physicians and solutions available. We need simple devices, cheaper equipments and user friendly solutions.

We have especially designed metallic conduits, patented them, used them in our institution to assess their utility, evaluated, and eliminated undesirable effects, corrected them in further improvements for the successful applications of any chest physician. Ours are well designed medical grade stainless steel conduits which can facilitate use of fiber optic bronchoscope for pleural visualization and interventions. Thus they combine the advantages of both rigid and flexible devices. It needs less manufacturing expertise, less engineering and craftsmanship resulting in reduction in costs. The training of physicians will be easier and cheaper making them user friendly. The procedure needs no sophisticated equipments, no major operation theater or no risky anesthesia.

\section{Devices}

There are three basic types of conduits-1) general utility 2) parietal and 3) visceral

\section{General description}

All these instruments are circular or oval cross sectioned high quality medical grade stainless tubes with detachable handles. They have smooth inner and outer surfaces and non-traumatic edges. They have a detachable handle of same material and fitting human ergonomics. The handle can be loosened and adjusted conveniently so that an assistant can hold the conduit in any desired position without moving. The set is provided with cleaning brushes so that internal cleaning is thorough.

A) Pleuroscopy facilitating conduit (Visceral): (Patent application No 3759/Mum/2013). This device is an angulated tubular structure useful for visualization of visceral pleura with a fiber optic bronchoscope. Its viewer-end has an air tube attached at right angle indicating direction of bevel of patient-end. There are two windows-one at the angulation on convex side, and other on the shaft both on same aspect. There is a free detachable curved handle with a clamp (Figure 1).

B) Parietal pleuroscopy facilitating conduit (introducer): (Patent application No 1066/ Mum/2012) this device is an angulated tubular

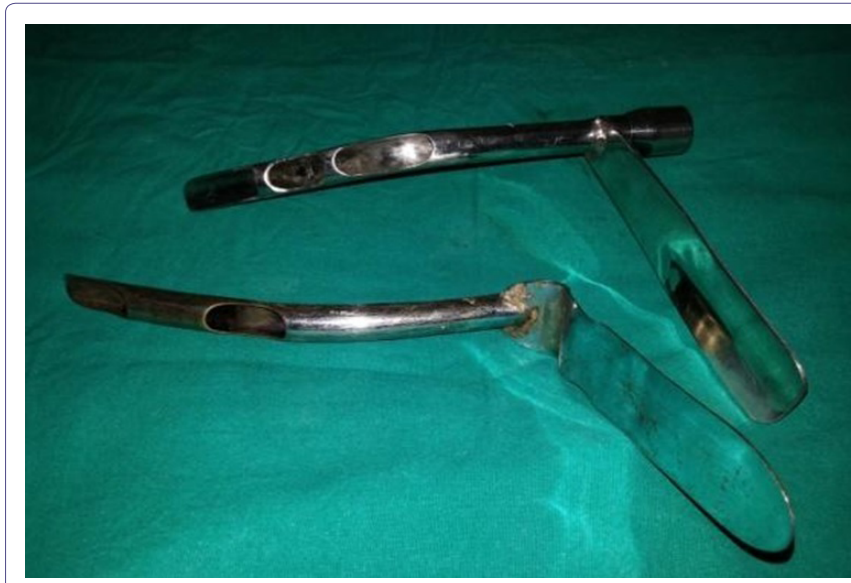

Figure 1: Pleuroscopy Facilitating Conduit (Visceral).

structure, useful for visualization of pleura with a fiber optic bronchoscope. Its viewer part has an air tube attached at right angle indicating the direction of beveled tip of patient part. There is window at the angulation, (Figure 2)

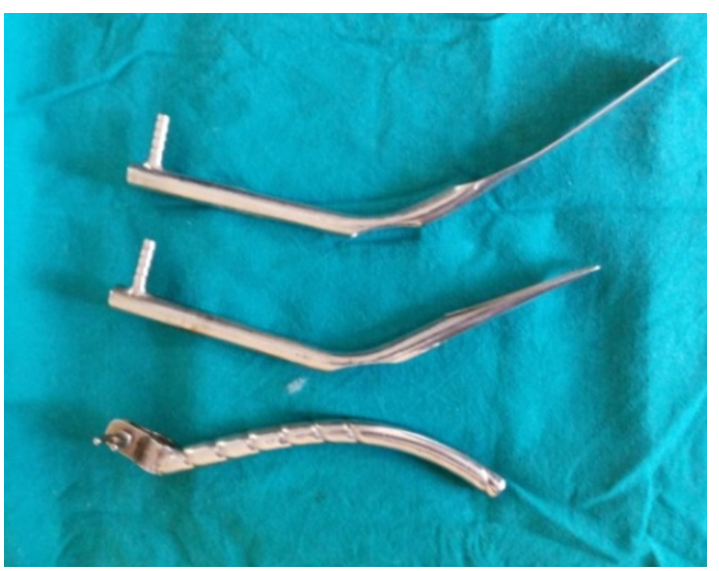

Figure 2: Parietal pleuroscopy facilitating conduits: Introducer and retractor.

C) The modification of this variety is called Parietal pleuroscopy facilitating device (retractor) (Patent application No 1400/ Mum/2012). Here the beveled end is replaced by a flat patula. This instrument will be useful in more invasive procedures to retract the lung.

D) General utility pleuroscopy conduits: These are metallic tubes with specified length and diameter with bevel. In straight kind the tube is straight, and in curved one it is smoothly curved. These conduits facilitate access to pleural cavity, drainage of pleural fluids and insertion of chest tubes.

\section{Aim and objectives}

Aim of this study was to assess the diagnostic and therapeutic utility of the conduits.

\section{Materials and Methods}

This is a retrospective serial study conducted in the respiratory ward of Department of Pulmonary Medicine, R.G. Gardi Medical College, Surasa, Ujjain MP and India from Jan 2014 to Dec 2019. 
Citation: Varudkar HG, Jain S, Julka A (2020) Diagnostic and Therapeutic Uses of A Novel Metallic Conduits Set and Fiber Optic Bronchoscope in Undiagnosed Pleural Diseases. J Pulm Med Respir Res 6: 045.

Ethics committee had approved it. Patients were included in this study with following inclusion criteria-Patients with ages more than $15 \mathrm{yrs}$; having undiagnosed moderate to massive pleural effusions, empyemas hemothorax or pneumothorax needing inter costal drainage and giving informed consent.

Exclusion criteria were: Unstable hemodynamics, HIV infection, bleeding tendencies, patients unable to give consent due to psychiatric illness, coma and language problems, and recent myocardial infarction. All patients underwent all routine diagnostic tests as per our hospital practice.- sputum examination for AFB, gram staining, pyogenic culture and sensitivity; serum protein, $\mathrm{LDH}$, blood sugar; Pleural fluid cytology and chemistry; pleural fluid microbiology: Gram stain, Z-N stain, fungal stain, pyogenic culture and sensitivity; and bronchoscopy in indicated cases. The patients whose etiological diagnosis remained unknown were included in the study. Patients with recurrent transudative fluid were also included for both diagnostic and therapeutic purposes.

Position was given to the patient depending on the comfort of the patients. Lateral recumbent or sitting or recumbent is acceptable. The desired area of chest was prepared and painted with denatured spirit and povidone iodine. The site if insertion, usually $5^{\text {th }}$ intercostal space midaxillary line, was anesthetized with the $2 \%$ lignocaine from skin, muscles up to pleura. A $2-2.5 \mathrm{~cm}$ long incision was made in skin and fascia; and a tract was made by separating the muscle layer with blunt dissection up to pleura, avoiding injuries to lung or diaphragm. Straight type of general utility pleuroscopy conduit can be used to facilitate the passage of chest tube. This gives steady drainage of fluid and keeps the stoma open. Fluid or air was allowed to drain in a gradual manner; and patient was allowed to settle for some time before the pleuroscopy was initiated.

After clinical assessment of vital parameters the procedure was started in lateral position with a sand bag below the chest to widen the intercostal spaces. Patient's upper arm was rested on head if arm rest was not available sometimes in minor operation room. Chest stoma created for chest tube insertion was used for procedure. Patient was cleaned and draped again. The desired conduit, especially parietal one was negotiated through the stoma and FOB was passed through the conduit. If the pleural space was not big enough then chances of lung damage are high. In such case tip of FOB was placed in the conduit and the conduit was passed under vision. Physician should just pass the chest wall thickness and slide the conduit parallel to it. Vertical thrust of conduit may lead to lung damage. The assistant holds the conduit in place. Schematic inspection of was done in following sequence: Apex, anterior, posterior and lateral surfaces of parietal pleura. To change the area of pleural surface the bronchoscope was withdrawn into the lumen of the conduit and then the conduit was moved to desirable place. Then the bronchoscope is advanced again to inspect newer pleural surface. The detachable handle can be loosened to adjust the direction of conduit and tightened again so that inconvenient position can be avoided. The parietal pleural conduit is then withdrawn after complete procedure.

For examination of lungs and visceral pleura the indigenous pleuroscopy facilitating device (visceral)- conduit is inserted. The window on convexity can give direct access to lungs. Otherwise negotiation to the complete lumen can give better lung vision. Inspection of visceral pleura was done systematically as described above. Relevant procedure such as pleural biopsy was done. Fiberoptic bronchoscope is withdrawn first followed by conduit. A chest tube was placed and under water seal was applied. Suitable stitches were applied. Dressing was done.

Following therapeutic interventions were done in appropriate cases

1. Limited adhesiolysis was done in cases of empyema mechanically with the instrument so that pus could be drained easily.

2. Positioning of chest tube was done in two cases where there was loculated fluid in follow up.

3. Retrieval of foreign body-A case of recurrent empyema was found to have synthetic non absorbable suture in pleural cavity. It was retrieved with foreign body forceps.

4. Pleurodesis was performed with Inj Oxytetracycline in oil $50 \mathrm{ml}$ was instilled with FOB in apical, anterior, posterior and diaphragmatic surfaces on lungs in 5 cases of recurrent pleural effusions. There was full lung expansion in follow up.

Appropriate antibiotics; pain killers and supportive care were given to all patients. Specific therapy for diagnosed disease e.g. anti-tubarcular therapy was initiated. Post-Operative Care was done in wards as usual. Usual daily care of chest tube i.e. observations of water column, drainage, administration of antibiotics, painkillers, nutrition and periodic radiological examinations were done. When there was drainage less than $50 \mathrm{ml} /$ day, closure of BPF, and full lung expansion on chest X-ray. The chest tube was removed with usual precautions.

\section{Observations and Discussion}

Out of 121 patients with pleural effusions, hydropneuthoraces or hemothoraces we could clinch diagnosis in 67 patients with routine investigations; but remaining 54 patients with undetermined diagnoses were subjected to pleuroscopy with our technique. Therefore analysis of these 54 patients is presented here. These 54 patients contained 33 males and 21 females. There were 18 patients (33\%) above age of 56 yrs, followed by $18(33 \%)$ in 36-45 years, and $16(30 \%)$ in $26-35 \mathrm{yrs}$ and 2 were still younger. 35 (65\%) patients had right sided, $13(25 \%)$ left and 6(10\%) bilateral pathology. Out of all patients 31 $(57 \%)$ had duration of symptoms less than 6 weeks and remaining 23 (43\%) had more prolonged illness, and 7 patients had recurrent history.

Pre procedure diagnoses of patients were 1) Penumothoraces 9 (17\%) 2) Pleural effusions-19 (35\%) 3) Hydropneumothoraces -11(20\%) 4) Empyema -15 (28\%). However hemorrhagic fluids were found in 9 patients at the time of initial diagnostic aspirations. Since analysis of pleural fluid could not clinch the diagnosis we had to opt for pleuroscopy in these patients. Out of 19 patients of pleural effusions, 6 patients had liver cirrhosis and 7 had chronic renal failure with transudative recurrent effusions. Remaining 6 had exudative effusions with indeterminate laboratory picture. The summary of all patients is given in table 1 which reveals that we have detected varied abnormalities.

In all 9 patients with pneumothoraces and 13 patients with hydrothoraces the pleurae were normal; but 4 patients had small blebs on visceral surfaces which required no interventions. Generalized redness with variable irregularities was found in 20 patients. Out of 
Citation: Varudkar HG, Jain S, Julka A (2020) Diagnostic and Therapeutic Uses of A Novel Metallic Conduits Set and Fiber Optic Bronchoscope in Undiagnosed Pleural Diseases. J Pulm Med Respir Res 6: 045.

\begin{tabular}{|c|c|c|c|c|}
\hline Sr no & Gross Appearance & No of Cases & Percent \% & $\begin{array}{l}\text { Histo pathology } \\
\text { Report }\end{array}$ \\
\hline 1 & Normal & 5 & 9 & Normal \\
\hline 2 & $\begin{array}{l}\text { Red- generalized or irregular } \\
\text { surface }\end{array}$ & $\begin{array}{c}12 \\
8\end{array}$ & $\begin{array}{l}23 \\
15\end{array}$ & $\begin{array}{l}\text { Inflammation } \\
\text { Tuberculosis }\end{array}$ \\
\hline 3 & Sub pleural blebs & 4 & 7 & Normal \\
\hline 4 & Pleural peel & 4 & 7 & Fibrosis \\
\hline 5 & Adhesions & $\begin{array}{l}8 \\
4\end{array}$ & $\begin{array}{c}15 \\
7\end{array}$ & $\begin{array}{l}\text { Fibrosis } \\
\text { Tuberculosis }\end{array}$ \\
\hline 6 & Tumors & 8 & 15 & $\begin{array}{c}\text { Mal. mesothelioma }=1 \\
\text { Adenacarcinoma }=2 \\
\text { Squamous cell carcinoma }=4 \\
\text { Anaplastic carcinoma }=1\end{array}$ \\
\hline 7 & Foreign body & 1 & 2 & Synthetic non absorbable thread \\
\hline & Total & 54 & 100 & \\
\hline
\end{tabular}

Table 1: Gross Appearances of Pleurae and Histopathology Reports.

them 8 patients had tuberculosis and rest, nonspecific inflammation. 12 patients with thin adhesions evaluated in laboratory showed tuberculosis in 4 and fibrosis in rest. Thus total $12(30 \%)$ patients with tuberculosis could be detected with pleuroscopy. V.K. Mootha, R. Agarwal, N. Singh, A.N. Aggarwal, et al [1-3] have reported diagnosis of Tuberculosis in 8 out of 35 patients $(22.9 \%)$ thoracoscopies. The difference may be due to many socioeconomic differences.

12 patients showed fibrosis in histopathology need some attention. Since these patients came to us late the original pathology remained a matter of conjuncture; but many of them can be due to tuberculosis which is common culprit in our patients. Had they come earlier we could have diagnosed more cases correctly.

8 patients (15\%) had malignancies one of them was malignant mesothelioma, a primary pleural tumor. However rest of them were secondaries. In the series of Randall J. H, Mani S. K, A. C. Mehta, et al [4] $27(66 \%)$ patients out of 47 had malignancies in undiagnosed patients. Thus we have fewer malignancies in our series

Sarkar SK [5] and his colleagues have already tried flexible fiber optic bronchoscope for diagnosis of 40 cases of pleural diseases got good results. However they have passed direct flexible fiber optic bronchoscopes in the pleurae without a proper conduit.

Davidson AC, George RJ, Sheldon CD [6], et al. have compared the results of rigid thoracoscopy with flexible fiber optic bronchoscope as thoracoscope in 30 patients. They did these procedures in bronchoscopy room; and their conclusion was that those procedures were safe and acceptable. However they found rigid thoracoscope better. The drawbacks they found have been obviated by our conduits which can guides the flexible scope to any desired site. Thus our techniques combine advantages of both rigid and flexible scopes.

Pleuroscopic examination was performed in 14 patients with the fiber optic bronchoscope by William $T$ and Thomas P [3] (5), and diagnosis was made in three. In the same series, out of 11 patients who remained undiagnosed even after blind needle biopsy, only three could be diagnosed by pleuroscopy with only FOB. Here again use of only FOB will not give satisfactory results.

It seems that with passage of time the outcome of flexible bronchoscope used as thoracoscope has not improved. In a recent series of undiagnosed 35 patients, reported by V.K. Mootha, R. Agarwal, N. Singh, et al [7], 5 patients remained undiagnosed with thoracoscopy. We have variety of cases; therefore comparison has limitations.

Thus use of our indigenous conduits along with fiber optic bronchoscopy is rewarding diagnostically

Therapeutic interventions

- Limited adhesiolysis was done in 3 cases of empyema mechanically with the instrument so that pus could be drained easily.

- Repositioning of chest tube was done in two cases where there was loculated fluid in follow up.

- Retrieval of foreign body-A case of recurrent empyema was found to have synthetic non-absorbable suture in pleural cavity. It was retrieved with foreign body forceps. (Figure 3)

- Pleurodesis -There was full lung expansion in follow up. Thus this instrument can be used for pleurodesis in cases of rapidly filling pleural effusion also.

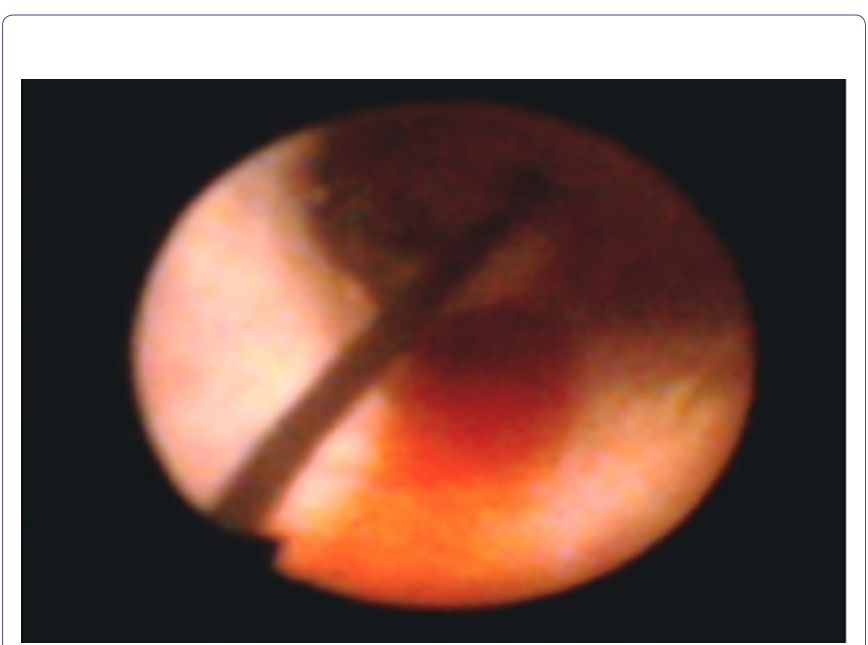

Figure 3: Foreign body. 
Shaw P and Agrawal R [8-10] have independently reviewed the results of 36 RCTs on 1499 subjects with malignant pleural effusions and concluded that chemical sclerosants have given significantly better results than control. Thus some therapeutic interventions are possible in selected cases.

\section{Complications}

- Bleeding - five patients of malignant effusions. It was not significant did not require any other intervention or blood transfusion.

- Hypoxia during the procedure occurred in 3 patients with bronchopleural fistula as they developed cough. However allowing the cough settle with some changes in posture, and oxygen inhalation was enough to correct hypoxia

- Pain during the procedure- 7 patients had severe pain during the procedure but sedation with Midazolam was given and the procedure could be completed. The pain not responding to above measures was probably due to pressure of conduit on intercostal nerve. This will be rectified by reducing the outer diameter in future.

There are many major complications associated with VATS [8] which are totally bypassed in our procedure. General anesthesia related complications are many; so are with thoracoscopes e. g. inadvertent insertion of trocar below the diaphragm or damage to the lungs, Equipment malfunction, Jamming or breaking of endo-staplers, etc. Barring the last, all of the complications are procedure related; thus conduits were safe to use

\section{Conclusion}

We have designed, developed, patented and tried two basic varieties of conduits for use of fiber optic bronchoscope for medical thoracoscopy. Parietal variety is primarily more useful for parietal pleura and the visceral one for visceral layer. There are some sub varieties of each prototype which can be used for more procedures during interventional thoracoscopy. By using this technique, pleuroscopy was done in undiagnosed 54 patients with - Penumothoraces - 9 (17\%) 2) Pleural effusions-19 (35\%) 3) Hydropneuthoraces -11(20\%) 4) Empyema $-15(28 \%)$ in our department. The procedure was done in two stages under local anesthesia sedation and painkillers. Stage 1- was drainage of pleural contents with chest tube, and Stage 2, pleuroscopy through the conduits.

Histopathology showed that there were 12 cases of tuberculosis and 8 cases of malignancies. This suggests that our procedure has given good diagnostic yield. Retrieval of Foreign body, adhesiolysis, pleurodesis, repositioning of chest tubes, etc therapeutic procedures could be done easily without conventional scopes.

There were complications like minimal bleeds, pain, and correctable hypoxia. However these are unrelated to instrumentation. Neural pain is one complication which is related to the diameter of instrument; but by its reduction also will reduce the pain. Corrections will be done in newer versions.

Thus over all this procedure is found to be easy, safe, acceptable to the patients with usual complications. The diagnostic yield is comparable to conventional procedures. It has also helped in cost reduction.

\section{Acknowledgement}

Mr. Anant Kshirsagar, an engineer in Pune helped in manufacturing the conduits; and Dr V.K Mahadik, Medical director, R.D Gardi Medical College, Ujjain permitted us to use in the patients

\section{References}

1. Ben-Isaac FB, Simmons DH (1975) Flexible fiber optic pleuroscopy: Pleural and lung biopsy. Chest 67: 573-576.

2. RW Light (2006) Parapneumonic Effusions and Empyema. Proc Am Thorac Soc 3: 75-80.

3. Mootha VK, Agarwal R, Singh N, Aggarwal AN, Gupta D, et al. (2011) Medical thoracoscopy for undiagnosed pleural effusions: Experience from a tertiary care hospital in North India. The Indian Journal of Chest Diseases \& Allied Sciences 53: 192.

4. Randall JH, Mani SK, Mehta AC, Medendorp SV, Wiedemann HP, et al. (1995) The impact of thoracoscopy on the management of pleural disease. Chest 107: 845-852.

5. Sarkar SK, Purohit SD, Sharma TN, et al. (1985) Pleuroscopy in the diagnosis of pleural effusions using flexible fiber optic bronchoscopes. Tubercle 66: 141-144.

6. Davidson AC, George RJ, Sheldon CD, Sinha G, Corrin B, et al. (1988) Thoracoscopy: Assessment of a physician service and comparison of a flexible bronchoscope used as a thoracoscope with a rigid thoracoscope. Thorax 43: 327-332.

7. Williams T, Thomas P (1981) The diagnosis of pleural effusions by fiberoptic bronchoscopy and pleuroscopy. Chest 80: 566-569.

8. Shaw P, Agrawal R (1996) Pleurodesis for malignant pleural effusions. Cochrane Database Syst Rev 14: 43.

9. Herrington JD, Gora-Harper ML, Salley RK (1996) Chemical pleurodesis with doxycycline $1 \mathrm{~g}$. Pharmacotherapy 16: 280-285.

10. Kumar DR (2001) complications and limitations of video assisted thoracic surgery. Current Medical Trends 5: 946-950. 


\section{H}

Advances In Industrial Biotechnology | ISSN: 2639-5665

Advances In Microbiology Research | ISSN: 2689-694X

Archives Of Surgery And Surgical Education | ISSN: 2689-3126

Archives Of Urology

Archives Of Zoological Studies | ISSN: 2640-7779

Current Trends Medical And Biological Engineering

International Journal Of Case Reports And Therapeutic Studies | ISSN: 2689-310X

Journal Of Addiction \& Addictive Disorders | ISSN: 2578-7276

Journal Of Agronomy \& Agricultural Science | ISSN: 2689-8292

Journal Of AIDS Clinical Research \& STDs | ISSN: 2572-7370

Journal Of Alcoholism Drug Abuse \& Substance Dependence | ISSN: 2572-9594

Journal Of Allergy Disorders \& Therapy | ISSN: 2470-749X

Journal Of Alternative Complementary \& Integrative Medicine | ISSN: 2470-7562

Journal Of Alzheimers \& Neurodegenerative Diseases | ISSN: 2572-9608

Journal Of Anesthesia \& Clinical Care | ISSN: 2378-8879

Journal Of Angiology \& Vascular Surgery | ISSN: 2572-7397

Journal Of Animal Research \& Veterinary Science | ISSN: 2639-375

Journal Of Aquaculture \& Fisheries | ISSN: 2576-5523

Journal Of Atmospheric \& Earth Sciences | ISSN: 2689-8780

Journal Of Biotech Research \& Biochemistry

Journal Of Brain \& Neuroscience Research

Journal Of Cancer Biology \& Treatment | ISSN: 2470-7546

Journal Of Cardiology Study \& Research | ISSN: 2640-768X

Journal Of Cell Biology \& Cell Metabolism | ISSN: 2381-1943

Journal Of Clinical Dermatology \& Therapy | ISSN: 2378-8771

Journal Of Clinical Immunology \& Immunotherapy | ISSN: 2378-8844

Journal Of Clinical Studies \& Medical Case Reports | ISSN: 2378-880

Journal Of Community Medicine \& Public Health Care | ISSN: 2381-1978

Journal Of Cytology \& Tissue Biology | ISSN: 2378-9107

Journal Of Dairy Research \& Technology | ISSN: 2688-9315

Journal Of Dentistry Oral Health \& Cosmesis | ISSN: 2473-6783

Journal Of Diabetes \& Metabolic Disorders | ISSN: 2381-201X

Journal Of Emergency Medicine Trauma \& Surgical Care | ISSN: 2378-8798

Journal Of Environmental Science Current Research | ISSN: 2643-5020

Journal Of Food Science \& Nutrition | ISSN: 2470-1076

Journal Of Forensic Legal \& Investigative Sciences | ISSN: 2473-733X

Journal Of Gastroenterology \& Hepatology Research | ISSN: 2574-2566
Journal Of Genetics \& Genomic Sciences | ISSN: 2574-2485

Journal Of Gerontology \& Geriatric Medicine | ISSN: 2381-8662

Journal Of Hematology Blood Transfusion \& Disorders | ISSN: 2572-2999

Journal Of Hospice \& Palliative Medical Care

Journal Of Human Endocrinology | ISSN: 2572-9640

Journal Of Infectious \& Non Infectious Diseases | ISSN: 2381-8654

Journal Of Internal Medicine \& Primary Healthcare | ISSN: 2574-2493

Journal Of Light \& Laser Current Trends

Journal Of Medicine Study \& Research | ISSN: 2639-5657

Journal Of Modern Chemical Sciences

Journal Of Nanotechnology Nanomedicine \& Nanobiotechnology | ISSN: 2381-2044

Journal Of Neonatology \& Clinical Pediatrics | ISSN: 2378-878X

Journal Of Nephrology \& Renal Therapy | ISSN: 2473-7313

Journal Of Non Invasive Vascular Investigation | ISSN: 2572-7400

Journal Of Nuclear Medicine Radiology \& Radiation Therapy | ISSN: 2572-7419

Journal Of Obesity \& Weight Loss | ISSN: 2473-7372

Journal Of Ophthalmology \& Clinical Research | ISSN: 2378-8887

Journal Of Orthopedic Research \& Physiotherapy | ISSN: 2381-2052

Journal Of Otolaryngology Head \& Neck Surgery | ISSN: 2573-010X

Journal Of Pathology Clinical \& Medical Research

Journal Of Pharmacology Pharmaceutics \& Pharmacovigilance | ISSN: 2639-5649

Journal Of Physical Medicine Rehabilitation \& Disabilities | ISSN: 2381-8670

Journal Of Plant Science Current Research | ISSN: 2639-3743

Journal Of Practical \& Professional Nursing | ISSN: 2639-568

Journal Of Protein Research \& Bioinformatics

Journal Of Psychiatry Depression \& Anxiety | ISSN: 2573-0150

Journal Of Pulmonary Medicine \& Respiratory Research | ISSN: 2573-0177

Journal Of Reproductive Medicine Gynaecology \& Obstetrics | ISSN: 2574-2574

Journal Of Stem Cells Research Development \& Therapy | ISSN: 2381-2060

Journal Of Surgery Current Trends \& Innovations | ISSN: 2578-7284

Journal Of Toxicology Current Research | ISSN: 2639-3735

Journal Of Translational Science And Research

Journal Of Vaccines Research \& Vaccination | ISSN: 2573-0193

Journal Of Virology \& Antivirals

Sports Medicine And Injury Care Journal | ISSN: 2689-8829

Trends In Anatomy \& Physiology | ISSN: 2640-7752

Submit Your Manuscript: https://www.heraldopenaccess.us/submit-manuscript 